University of Nebraska - Lincoln

DigitalCommons@University of Nebraska - Lincoln

July 1980

Phase transition effects: A crystallographic characterization of the temperature dependency of the crystal structure of the 1:1 charge transfer complex between anthracene and tetracyanobenzene in the temperature range 297 to $119 \mathrm{~K}$

John J. Stezowski

University of Nebraska - Lincoln, jjs@unlserve.unl.edu

Follow this and additional works at: https://digitalcommons.unl.edu/chemistrystezowski

Part of the Chemistry Commons

Stezowski, John J., "Phase transition effects: A crystallographic characterization of the temperature dependency of the crystal structure of the 1:1 charge transfer complex between anthracene and tetracyanobenzene in the temperature range 297 to 119 K" (1980). John J. Stezowski Publications. 3. https://digitalcommons.unl.edu/chemistrystezowski/3

This Article is brought to you for free and open access by the Published Research - Department of Chemistry at DigitalCommons@University of Nebraska - Lincoln. It has been accepted for inclusion in John J. Stezowski Publications by an authorized administrator of DigitalCommons@University of Nebraska - Lincoln. 


\title{
Phase transition effects: A crystallographic characterization of the temperature dependency of the crystal structure of the 1:1 charge transfer complex between anthracene and tetracyanobenzene in the temperature range 297 to $119 \mathrm{~K}$
}

\author{
John J. Stezowski \\ Institut für Organische Chemie, Biochemie und Isotopenforschung der Universität Stuttgart, \\ Pfaffenwaldring 55, D-7000 Stuttgart 80, Germany \\ (Received 7 August 1979; accepted 18 March 1980) \\ Crystal structures of the charge transfer complex between anthracene and tetracyanobenzene, A:TCNB, \\ $\mathrm{C}_{14} \mathrm{H}_{10}: \mathrm{C}_{10} \mathrm{H}_{2} \mathrm{~N}_{4}$, at three temperatures $(297,234$ and $226 \mathrm{~K}$ ) above the order-disorder phase transition \\ at $206 \mathrm{~K}$ and at four temperatures $(202,170,138$, and $119 \mathrm{~K})$ below it have been determined from \\ $\mathrm{x}$-ray diffraction data. The space group of the room temperature phase was assigned as $\mathrm{Cm}$ with \\ $a=9.528(2), b=12.779(3), c=7.441(2) \AA$ and $\beta=92.39(2)$ degrees at $297 \mathrm{~K}$ and for the low temperature \\ phase is $P 2_{1} /$ a with $a=9.457(1), b=12.689(2), c=7.325(1) \AA$ and $\beta=92.98(1)^{\circ}$ at $119 \mathrm{~K} ; Z=2$ in both \\ phases. The electron densities, plotted for both the donor and acceptor molecules at each temperature, \\ indicate that there is dynamic librational disorder in the $A$ molecules that gives rise to a single average \\ orientation in the room temperature phase. Below the phase transition, two temperature dependent, \\ symmetry related, orientations are observed; the libration diminishes slowly as a function of temperature \\ in this phase. There are also modest reorientations, relative to the room temperature phase, in the \\ TCNB molecules as the crystal is cooled. Crystal packing indicates that a network of intermolecular \\ dipole-dipole interactions between the heteroatomic acceptor molecules accounts for their lack of \\ libration comparable to that of the donors.
}

\section{INTRODUCTION}

The $1: 1$ charge transfer complex between anthracene, $A$, and tetracyanobenzene, TCNB, has been the subject of considerable recent interest, ${ }^{1-7}$ largely because of a phase transition at $206 \mathrm{~K}$. Interpretation of the spectroscopic data from the crystalline complex can be greatly facilitated by definitive structural data. The crystal structure of the A : TCNB complex at room temperature has been known for several years ${ }^{8}$ and a recent report from this laboratory briefly described the principal features of the structure at low temperature. ${ }^{9}$

Spectroscopic evidence indicates that the structure of the crystalline "low temperature" phase varies as a function of temperature. A report by Möhwald et al. ${ }^{3}$ demonstrated that the angle between the long axes of the donor molecules in the "low temperature" phase increased as the temperature decreased. That report prompted a decision to determine the structure of A : TCNB at several temperatures below the phase transition. The lack of definitive characterization of the disorder of the donor molecule in the room temperature phase as either clearly a case of statistical "static" disorder or dynamic disorder, prompted a further investigation of the system at three temperatures above the phase transition.

\section{EXPERIMENTAL}

All crystallographic measurements were made with the same crystal, a $0.48 \times 0.50 \times 0.65 \mathrm{~mm}^{3}$ parallelepiped cut from a larger plate. The crystal was enclosed in a thin walled glass capillary. Data were measured with a Syntex $\overline{\mathrm{Pl}}$ autodiffractometer equipped with a low temperature device (Syntex LT-1). After all crystallographic data had been collected, the respective temperatures were measured with an iron-constantan thermo- couple in the position previously occupied by the crystal. Lattice parameters, Table I, were determined by leastsquares refinement ${ }^{10}$ with automatically centered $2 \theta$ values for $\sim 50$ reflections in an angular range $30^{\circ}<2 \theta<46^{\circ}$ $\left(\operatorname{MoK}_{\alpha}, \lambda=0.71069 \AA\right)$.

Intensity data were measured in an $\omega$-scan mode with a scan range of $0.75^{\circ}$ and a variable scan rate, (Table II), determined by the maximum intensity of the reflection. Three reference reflections were monitored after each 200 data were measured. The maximum deviations from their average intensities and the resolution of the data sets are also presented in Table II; the sequence in which the various data sets were measured is also indicated therein. Data were corrected for Lorentz and polarization effects. The absorption coefficient $\mu=0.9$ $\mathrm{cm}^{-1}$ gives rise to minimum and maximum absorption corrections of 1.04 and 1.06 , respectively; absorption corrections were deemed unnecessary.

\section{STRUCTURE DETERMINATION AND REFINEMENT}

The coordinates reported by Tsuchiya et $a l .{ }^{8}$ for the crystal structure of A : TCNB at room temperature were used for the initial model, as was their suggestion that the space group is $C m$. Refinement was effected by block diagonal least-squares techniques in which the blocks contained the variables of 2 or $3 \mathrm{C}$ or $\mathrm{N}$ atoms and those of any $\mathrm{H}$ atoms bonded to them. A single scale factor was refined with each data set; all appropriate fractional atomic coordinates, anisotropic temperature factors for $\mathrm{C}$ and $\mathrm{N}$ atoms, and isotropic temperature factors for $\mathrm{H}$ atoms were refined. The same weighting scheme was used in each refinement: $w=1 / \sigma^{2}=\left\{\sigma^{2}\left(F_{0}\right)\right.$ $\left.+0.0125\left|F_{0}\right|+0.0005\left|F_{0}\right|^{2\}}\right\}^{-1}$. The possibility of the correct space group being centrosymmetric $(\mathrm{C} 2 / \mathrm{m})$ was tested for the $226 \mathrm{~K}$ data set. The resultant $R_{w}$ value, 
TA BLE I. Lattice parameters and unit cell volume at the indicated temperatures.

\begin{tabular}{llllll}
\hline \hline$T(\mathrm{~K})$ & $a(\AA)$ & $b(\AA)$ & $c(\AA)$ & $\beta(\mathrm{deg})$ & $V\left(\AA^{3}\right)$ \\
\hline $297(2)$ & $9.528(2)$ & $12.779(3)$ & $7.441(2)$ & $92.39(2)$ & 905.22 \\
$234(2)$ & $9.515(3)$ & $12.736(5)$ & $7.391(2)$ & $92.53(3)$ & 894.79 \\
$226(2)$ & $9.519(3)$ & $12.730(5)$ & $7.384(2)$ & $92.57(3)$ & 893.93 \\
$202(2)$ & $9.504(2)$ & $12.733(3)$ & $7.379(1)$ & $92.72(2)$ & 891.99 \\
$170(2)$ & $9.484(1)$ & $12.722(2)$ & $7.366(1)$ & $92.80(1)$ & 887.63 \\
$138(2)$ & $9.462(1)$ & $12.695(2)$ & $7.334(1)$ & $92.96(1)$ & 879.79 \\
$119(2)$ & $9.457(1)$ & $12.689(2)$ & $7.325(1)$ & $92.98(1)$ & 877.77 \\
\hline
\end{tabular}

0.101 , when compared with that from space group $\mathrm{Cm}$, 0.098 , gives a level of confidence of $\sim 70 \%$ that the crystal structure is noncentrosymmetric. ${ }^{18}$ The selection of the noncentrosymmetric space group should not be taken as definitive. No physical measurements were conducted to attempt to establish the absence of a crystallographic inversion center. The thermal parameters for the A molecules were similar for the refinements in both space groups so the question of the presence of the inversion center in the room temperature phase is not crucial to this study. The conventional residuals are presented in Table II, atomic coordinates in Table III. Temperature factors and calculated and observed structure factors have been deposited. ${ }^{11}$

\section{DISCUSSION}

\section{Characterization of the disorder}

Disorder in the solid state is generally characterized by either of two extremes, statistical "static" disorder which is describable as a double well potential with $E_{a}$ $\gg k T$ or as dynamic disorder for which the energy diagram displays a flat bottomed well. Systems which undergo an "order-disorder" phase transition are characterizable with a combination of single and double well potentials.

Statistical "static" disorder can often be characterized by a crystal structure analysis. In such cases, the individual sites are far enough apart to be unequivocally resolved by the resolution of the data set (see, for example Ref. 12). Unfortunately, this is frequently not the case when disorder is encountered in charge transfer complexes. Attempts to characterize disorder crystallographically in such cases usually depends on the analysis of difference Fourier maps or on rigid body thermal motion analysis. In both cases, limitations of the structural model severely hinder definitive characterization. In the first method, inadequacies of the model introduce errors in both the $|\Delta F|$ and in the phases. The second technique is limited by the fact that the quality of the description of thermal motion with elliptical anisotropic temperature factors is inversely proportional to the magnitude of the motion in the crystal. This study has addressed the characterization of the disorder in the A : TCNB complex by determining the crystal structure at several temperatures in order to examine the nature of the potential wells governing the donor and acceptor molecules therein.

Electron density maps, $F_{0}$ Fourier syntheses, calculated with the phases from the fully refined structural models at each temperature provide a method for exam-

TABLE II. Parameters characterizing the data collection and refinement of the A: TCNB crystal structure determination.

\begin{tabular}{|c|c|c|c|c|c|c|c|}
\hline$T(\mathrm{~K})$ & $297^{2}$ & $234^{\mathrm{a}}$ & $226^{\mathrm{a}}$ & $202^{b}$ & $170^{\mathrm{b}}$ & $138^{b}$ & $119^{\mathrm{b}}$ \\
\hline Data set No. & 2 & 5 & 4 & 3 & 7 & 6 & 1 \\
\hline Scan speed $(\% / \mathrm{min})$ & $2.0-24.0$ & $3.0-24.0$ & $3.0-24.0$ & $3.0-24.0$ & $3.0-24.0$ & $3.0-24.0$ & $3.0-24.0$ \\
\hline $\begin{array}{l}\text { Resolution }{ }^{c} \\
2 \theta_{\max }(\mathrm{deg})\end{array}$ & 70 & 60 & 60 & 60 & 70 & 70 & 70 \\
\hline $\begin{array}{l}\text { Max. dev. in } I \\
\text { refinement }\end{array}$ & $2.2 \%$ & $0.8 \%$ & $1.8 \%$ & $0.5 \%$ & $3.1 \%$ & $4.8 \%$ & $4.3 \%$ \\
\hline $\begin{array}{l}\text { No. } h k l \text { 's } \\
\text { measured }\end{array}$ & 2067 & 1364 & 1357 & 2601 & 3903 & 3876 & 3853 \\
\hline No. $h k l$ 's observed & 1303 & 1103 & 1092 & 1745 & 2332 & 2626 & 3156 \\
\hline$R$ & 0.054 & 0.052 & 0.057 & 0.070 & 0.052 & 0.045 & 0.053 \\
\hline$R_{w}$ & 0,089 & 0.075 & 0.092 & 0.108 & 0.075 & 0.063 & 0.086 \\
\hline$\Sigma^{\mathrm{d}}$ & 1.3 & 1.3 & 1.5 & 1.6 & 1.3 & 1.2 & 1.3 \\
\hline $\begin{array}{l}\left|F_{0}\right|_{\max } \\
(h+k=2 n)\end{array}$ & 166 & 163 & 179 & 172 & 152 & 154 & 213 \\
\hline $\begin{array}{l}\left|F_{0}\right|_{\max }^{1} \\
(h+k=2 n+1)\end{array}$ & $\cdots$ & $\cdots$ & $\cdots$ & 13 & 18 & 21 & 22 \\
\hline
\end{tabular}

aspace group $C 2 / m, C m$ or $C 2[C m$ used in refinement (8)].

Space group $P 2_{1} / a$.

${ }^{c}{ }_{M \circ} K_{\alpha}$ radiation $\lambda=0.71069 \AA$.

${ }^{\mathrm{d}}$ Estimated standard deviation in an observation of unit weight.

$\left|F_{0}\right|_{\text {min }}$ for observed reflections is $\sim 1$ at each temperature.

'Systematically extinct reflections were not measured. 
TABLE III. Fractional atomic coordinates with estimated standard deviations.

\begin{tabular}{|c|c|c|c|c|c|c|c|}
\hline & & & & \\
\hline & & & & & $10^{4} x$ & $10^{4} y$ & $10^{4} z$ \\
\hline & $10^{4} x$ & $10^{4} y$ & $10^{4} z$ & \multirow{2}{*}{\multicolumn{4}{|c|}{$T=170 \mathrm{~K}$}} \\
\hline$T=297 \mathrm{~K}$ & & & & & & & \\
\hline $\mathrm{Cl}$ & $-109(7)$ & $1084(2)$ & $15(9)$ & $\mathrm{C} 1$ & $-150(1)$ & $1092(1)$ & $75(1)$ \\
\hline $\mathrm{C} 2$ & $1099(3)$ & $541(3)$ & $-582(3)$ & $\mathrm{C} 2$ & $1104(1)$ & $667(1)$ & $-509(1)$ \\
\hline C3 & $2326(5)$ & $1107(6)$ & $-1109(5)$ & $\mathrm{C} 3$ & $2234(1)$ & $1315(1)$ & $-1049(2)$ \\
\hline $\mathrm{C} 4$ & $3468(5)$ & $581(9)$ & $-1642(5)$ & $\mathrm{C} 4$ & $3448(1)$ & $896(2)$ & $-1605(2)$ \\
\hline C5 & $-1256(3)$ & $538(3)$ & $567(3)$ & C5 & $-1261(1)$ & $454(1)$ & $587(1)$ \\
\hline $\mathrm{C} 6$ & $-2467(6)$ & $1048(7)$ & $1163(7)$ & $\mathrm{C} 6$ & $-2556(1)$ & $865(1)$ & $1211(2)$ \\
\hline $\mathrm{C} 7$ & $-3563(3)$ & $499(9)$ & $1668(5)$ & $\mathrm{C} 7$ & $-3614(1)$ & $201(2)$ & $1691(2)$ \\
\hline C 8 & $-44(5)$ & $1098(2)$ & $5006(8)$ & $\mathrm{C} 8$ & $-39(1)$ & $1106(1)$ & $4976(1)$ \\
\hline $\mathrm{c} 9$ & $1115(2)$ & $555(2)$ & $4513(3)$ & $\mathrm{C} 9$ & $1169(1)$ & $573(1)$ & $4490(1)$ \\
\hline C10 & $2352(2)$ & $1122(2)$ & $4002(3)$ & $\mathrm{C} 10$ & $2378(1)$ & $1159(1)$ & $3952(1)$ \\
\hline $\mathrm{C} 11$ & $-1246(2)$ & $542(2)$ & $5510(3)$ & $\mathrm{C} 11$ & $-1206(1)$ & $535(1)$ & $5484(1)$ \\
\hline $\mathrm{C} 12$ & $-2473(2)$ & $1101(2)$ & $6024(4)$ & $\mathrm{C} 12$ & $-2464(1)$ & $1079(1)$ & $5970(1)$ \\
\hline N1 & $3321(3)$ & $1566(2)$ & $3563(4)$ & N1 & $3333(1)$ & $1633(1)$ & $3511(1)$ \\
\hline $\mathrm{N} 2$ & $-3440(3)$ & $1567(2)$ & $6398(4)$ & N2 & $-3464(1)$ & $1523(1)$ & $6340(2)$ \\
\hline \multicolumn{4}{|l|}{$T=234 \mathrm{~K}$} & \multicolumn{4}{|l|}{$T=138 \mathrm{~K}$} \\
\hline$C 1$ & $-99(7)$ & $1095(2)$ & $25(7)$ & $\mathrm{C} 1$ & $-169(1)$ & $1094(1)$ & $96(1)$ \\
\hline C2 & $1101(2)$ & $571(2)$ & $-527(3)$ & $\mathrm{C} 2$ & $1097(1)$ & $682(1)$ & $-500(1)$ \\
\hline $\mathrm{C} 3$ & $2327(4)$ & $1113(4)$ & $-1108(4)$ & $\mathrm{C3}$ & $2219(1)$ & $1345(1)$ & $-1032(1)$ \\
\hline $\mathrm{C} 4$ & $3471(4)$ & $589(6)$ & $-1629(5)$ & $\mathrm{C} 4$ & $3446(1)$ & $930(1)$ & $-1604(1)$ \\
\hline $\mathrm{C} 5$ & $-1257(2)$ & $548(2)$ & $564(3)$ & C5 & $-1272(1)$ & $441(1)$ & $596(1)$ \\
\hline C6 & $-2481(4)$ & $1062(5)$ & $1149(5)$ & $\mathrm{C} 6$ & $-2572(1)$ & $842(1)$ & $1224(1)$ \\
\hline c7 & $-3589(3)$ & $519(5)$ & $1648(4)$ & $\mathrm{C} 7$ & $-3624(1)$ & $173(1)$ & $1701(1)$ \\
\hline $\mathrm{C} 8$ & $-36(5)$ & $1102(1)$ & $5008(6)$ & $\mathrm{C} 8$ & $-42(1)$ & $1108(1)$ & $4980(1)$ \\
\hline $\mathrm{C} 9$ & $1119(2)$ & $558(2)$ & $4517(3)$ & $\mathrm{C} 9$ & $1169(1)$ & $578(1)$ & $4492(1)$ \\
\hline $\mathrm{C} 10$ & $2356(2)$ & $1123(2)$ & $3997(3)$ & $\mathrm{C} 10$ & $2376(1)$ & $1164(1)$ & $3950(1)$ \\
\hline $\mathrm{Cl1}$ & $-1251(2)$ & $549(2)$ & $5511(3)$ & $\mathrm{C} 11$ & $-1209(1)$ & $531(1)$ & $5485(1)$ \\
\hline $\mathrm{C} 12$ & $-2472(2)$ & $1107(2)$ & $6025(3)$ & $\mathrm{C} 12$ & $-2474(1)$ & $1075(1)$ & $5963(1)$ \\
\hline $\mathrm{N} 1$ & $3328(2)$ & $1574(2)$ & $3567(3)$ & $\mathrm{N} 1$ & $3333(1)$ & $1643(1)$ & $3504(1)$ \\
\hline N2 & $-3443(2)$ & $1574(2)$ & $6402(3)$ & $\mathrm{N} 2$ & $-3477(1)$ & $1516(1)$ & $6332(1)$ \\
\hline \multicolumn{4}{|l|}{$T=226 \mathrm{~K}$} & \multicolumn{4}{|l|}{$T=119 \mathrm{~K}$} \\
\hline $\mathrm{Cl}$ & $-64(6)$ & $1092(2)$ & $7(7)$ & $\mathrm{C} 1$ & $-178(1)$ & $1099(1)$ & $107(1)$ \\
\hline $\mathrm{C}^{2}$ & $1128(3)$ & $579(3)$ & $-533(3)$ & $\mathrm{C} 2$ & $1095(1)$ & $688(1)$ & $-494(1)$ \\
\hline $\mathrm{C} 3$ & $2368(5)$ & $1095(5)$ & $-1110(5)$ & $\mathrm{C} 3$ & $2211(1)$ & $1364(1)$ & $-1021(2)$ \\
\hline $\mathrm{C} 4$ & $3528(4)$ & $590(8)$ & $-1618(5)$ & $\mathrm{C} 4$ & $3449(1)$ & $951(1)$ & $-1598(2)$ \\
\hline $\mathrm{C} 5$ & $-1233(3)$ & $541(3)$ & $563(3)$ & $\mathrm{C} 5$ & $-1279(1)$ & $433(1)$ & $601(1)$ \\
\hline $\mathrm{C} 6$ & $-2442(6)$ & $1086(6)$ & $1152(6)$ & $\mathrm{C} 6$ & $-2579(1)$ & $833(1)$ & $1233(2)$ \\
\hline $\mathrm{C} 7$ & $-3538(3)$ & $514(7)$ & $1673(5)$ & $\mathrm{C} 7$ & $-3638(1)$ & $158(2)$ & $1708(2)$ \\
\hline $\mathrm{C} 8$ & $-17(4)$ & $1107(2)$ & $5003(6)$ & $\mathrm{C} 8$ & $-41(1)$ & $1112(1)$ & $4984(1)$ \\
\hline $\mathrm{C} 9$ & $1158(2)$ & $566(2)$ & $4514(3)$ & C9 & $1171(1)$ & $578(1)$ & $4495(1)$ \\
\hline $\mathrm{C} 10$ & $2393(3)$ & $1114(2)$ & $3995(4)$ & $\mathrm{C} 10$ & $2374(1)$ & $1170(1)$ & $3956(1)$ \\
\hline $\mathrm{C} 11$ & $-1205(2)$ & $536(2)$ & $5508(3)$ & $\mathrm{C} 11$ & $-1214(1)$ & $531(1)$ & $5486(1)$ \\
\hline $\mathrm{C} 12$ & $-2440(2)$ & $1120(2)$ & $6011(4)$ & $\mathrm{C} 12$ & $-2476(1)$ & $1076(1)$ & $5963(2)$ \\
\hline $\mathrm{N} 1$ & $3381(3)$ & $1575(2)$ & $3585(4)$ & N1 & $3340(1)$ & $1647(1)$ & $3502(2)$ \\
\hline $\mathrm{N}^{2}$ & $-3394(3)$ & $1570(2)$ & $6411(4)$ & N2 & $-3490(1)$ & $1517(1)$ & $6330(2)$ \\
\hline
\end{tabular}

TABLE III (Continued)

\begin{tabular}{lrrr}
$T=202 \mathrm{~K}$ & & & \\
C1 & $-95(2)$ & $1093(2)$ & $49(3)$ \\
C2 & $1132(2)$ & $633(2)$ & $-522(2)$ \\
C3 & $2289(3)$ & $1255(3)$ & $-1076(3)$ \\
C4 & $3465(3)$ & $803(4)$ & $-1618(2)$ \\
C5 & $-1231(2)$ & $490(2)$ & $574(2)$ \\
C6 & $-2513(3)$ & $920(3)$ & $1184(3)$ \\
C7 & $-3595(3)$ & $299(4)$ & $1681(3)$ \\
C8 & $-25(2)$ & $1105(1)$ & $4976(2)$ \\
C9 & $1175(2)$ & $566(1)$ & $4492(2)$ \\
C10 & $2390(2)$ & $1145(1)$ & $3959(3)$ \\
C11 & $-1198(2)$ & $541(1)$ & $5487(2)$ \\
C12 & $-2444(2)$ & $1092(1)$ & $5980(3)$ \\
N1 & $3347(2)$ & $1615(1)$ & $3529(3)$ \\
N2 & $-3432(2)$ & $1536(1)$ & $6361(3)$ \\
\hline
\end{tabular}

ining the structure that significantly reduces the effects of inadaquacies in the model. Electron density contours for the molecular planes of A and TCNB molecules are presented in Figs. 1 and 2, respectively, for each temperature studied. It is obvious from comparison of the figures that the A molecules are the most dramatically affected by cooling. The systematic changes in the plots in Fig. 1 are consistent with the description of the behavior of the donor molecules by a broad single well potential at temperatures above the phase transition at $T_{p}=206 \mathrm{~K}$ and by a series of double well potentials below $T_{p}$. The plots for the A molecules in the structure at $T>T_{p}$ show no indication of resolution of the atomic po- 
(a)

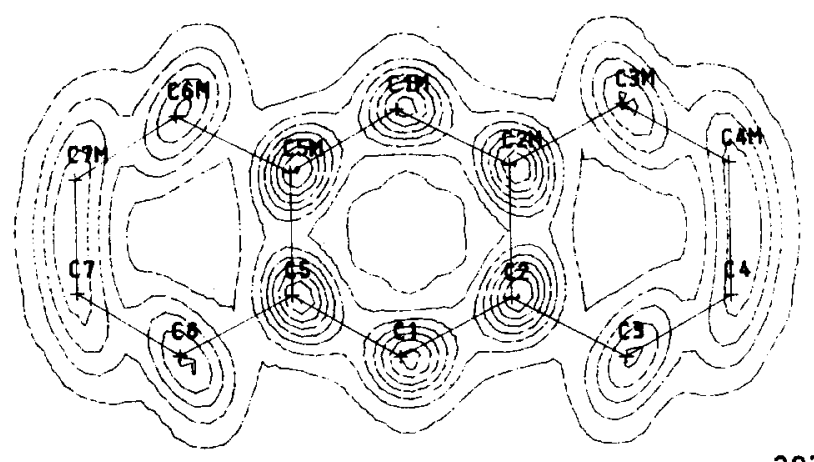

$297 K$
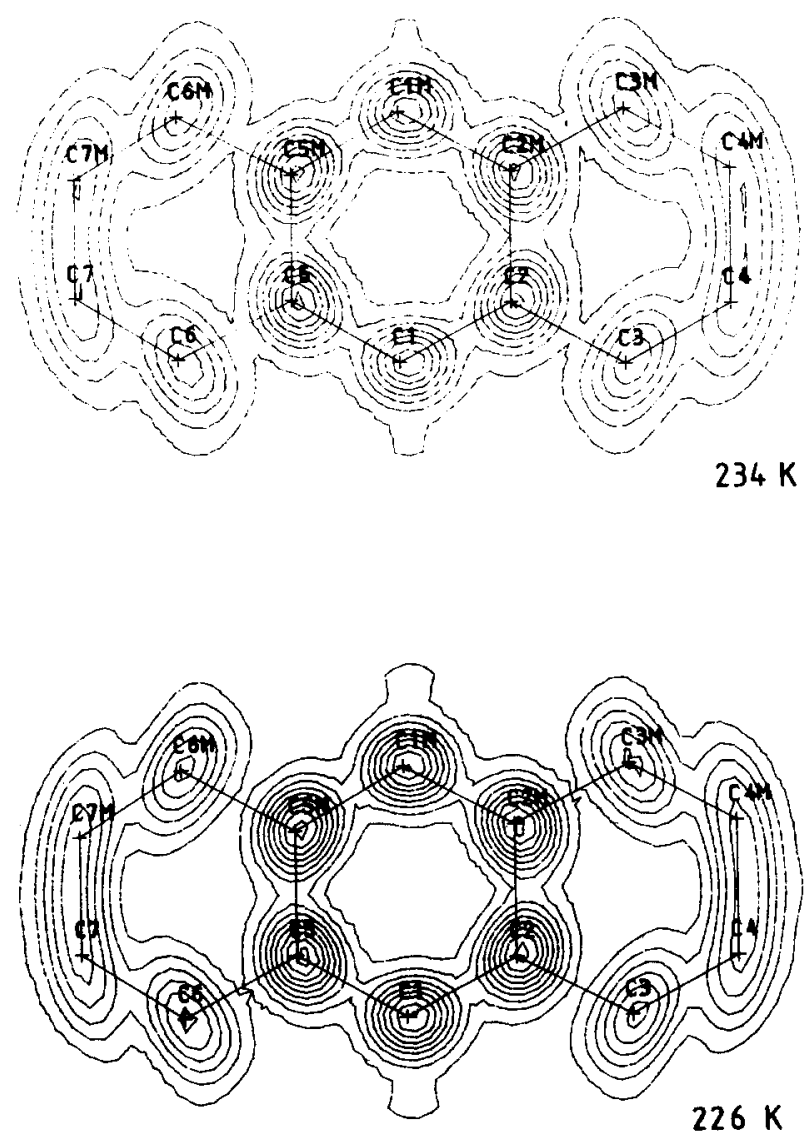

FIG. 1. Electron density, $F_{0}$, $\operatorname{plots}^{14}$ for the A molecules of $A:$ TCNB at the indicated temperatures. Part (a) contains examples of the room temperature phase, space group $\mathrm{Cm}$; and (b) displays examples from the low temperature phase, space group $P 2_{1} / a$. Plots are contoured at $1 \mathrm{e}^{-} \AA^{-3}$ levels. (b)

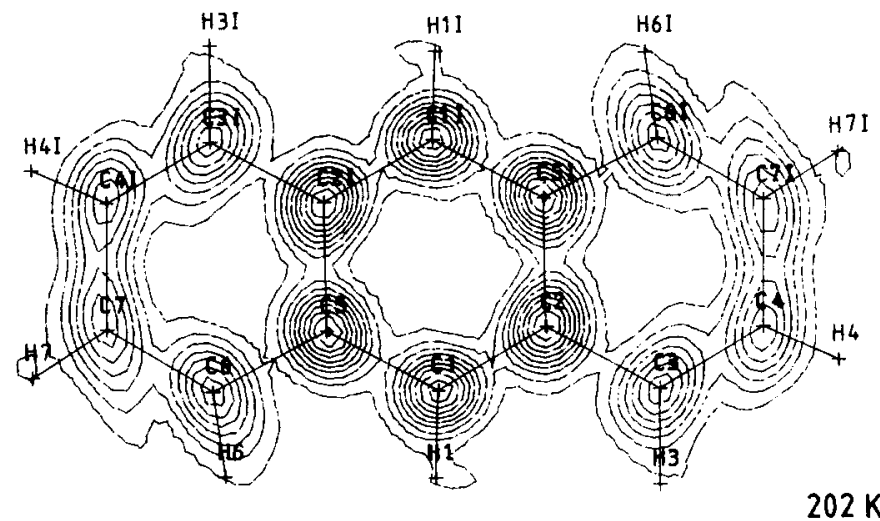

$202 \mathrm{~K}$
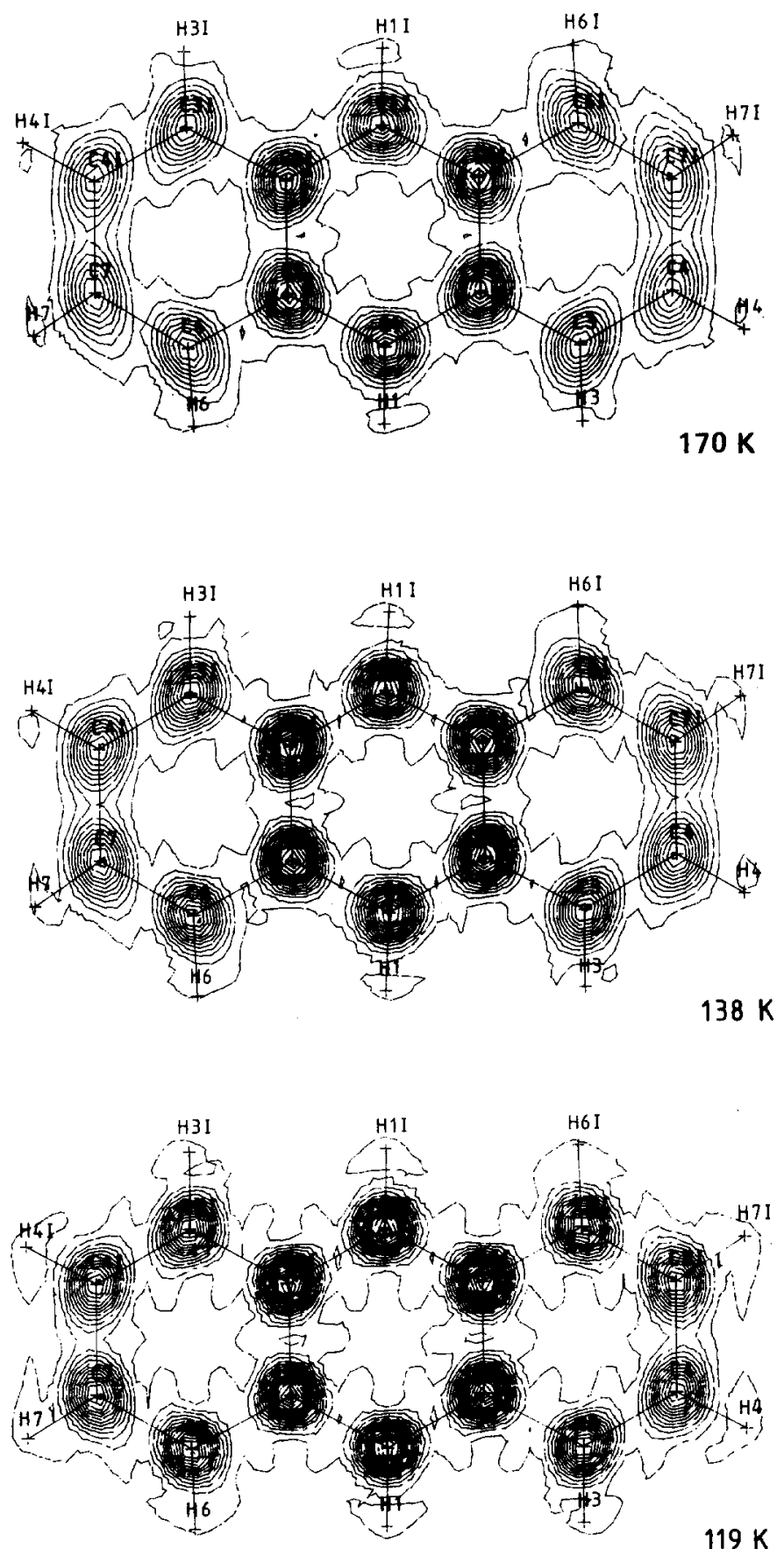
(a)
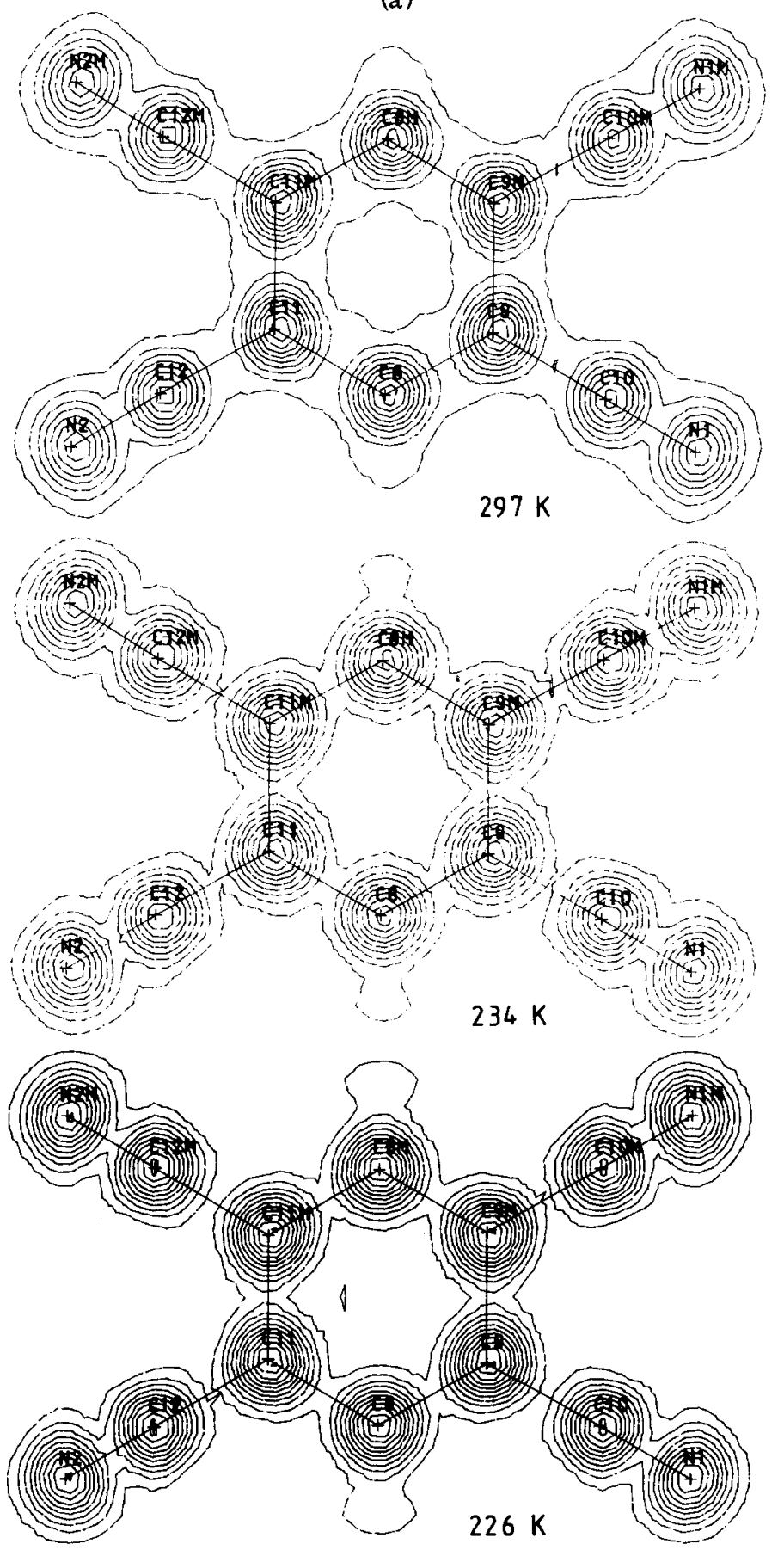

FIG. 2. Electron density $F_{0}$ plots $^{14}$ for the TCNB molecules of $A: T C N B$ at the indicated temperatures. Part (a) displays examples of the room temperature phase, and (b), of the low temperature phase. Plots are contoured at $1 \mathrm{e}^{-} \AA^{-3}$ levels. (b)
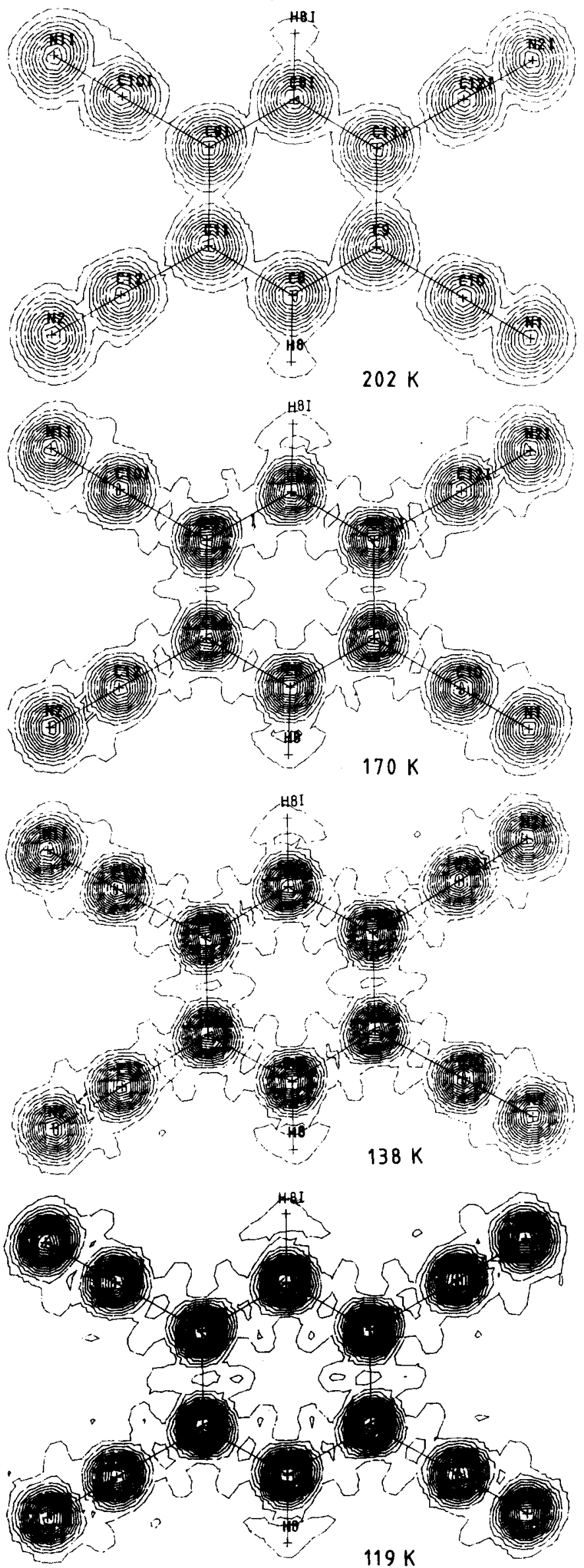


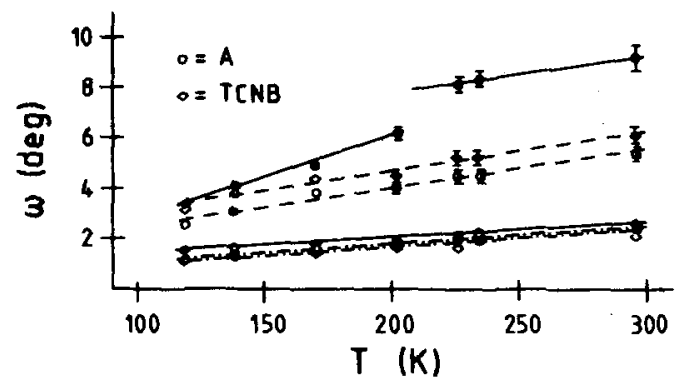

FIG. 3. The libration of A and TCNB molecules as a function of temperature. The magnitude $\omega$ of the libration about three principal axes was determined by rigid body thermal motion analysis. ${ }^{13}$ The direction of these axes correspond approximately with: - the normal to the molecular plane, - - the long axis of the respective molecule, and - - - the short molecular axis. The curve for the molecular libration about the plane normal of the A molecule (blackened circles) clearly shows a different slope in the room and low temperature phases. Errors in those values for which arror bars are not shown are on the order of magnitude of the size of the symbols.

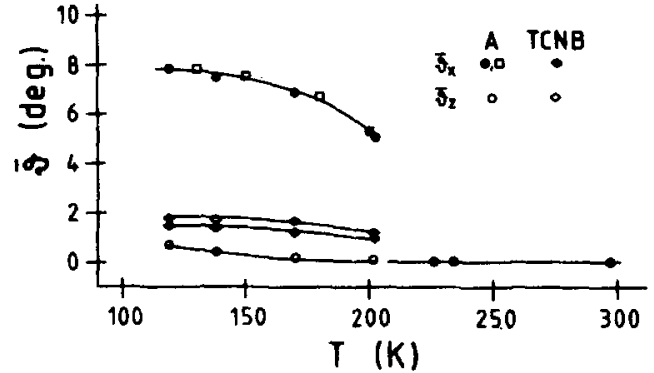

FIG. 4. The mean order parameter $\bar{\vartheta}_{x}=$ one-half the angle between the long axes of symmetry related molecules and $\bar{v}$. $=$ one-half the angle between plane normals of said molecules. The open squares represent points taken from the $\bar{\vartheta}_{x}$ curve determined by ESR techniques. ${ }^{3}$ Crystallographic symmetry requires that both $\bar{\vartheta}_{x}$ and $\mho_{z}$ be identically 0 in the room temperature phase. The points shown for this phase are intended only to indicate the temperature at which the structure was determined. Estimated standard deviations are approximately 0.15 deg or less.

TABLE IV. Least-squares planes and lines for A:TCNB components. The $i, j$, and $k$ axes of the orthogonal coordinate system are in directions: $a, b^{*}$, and $a \times b^{*}$.

(a). Least-squares planes of the form $p i+q j+r k=s$. All nonbydrogen atoms of A and TCNB were used to determine the planes by least-squares techniques. Sigma: is the standard deviation of the leastsquares plane determined from the distances of the atoms used to define the plane from the resultant plane.

\begin{tabular}{lllllllr} 
& $297 \mathrm{~K}$ & $234 \mathrm{~K}$ & $226 \mathrm{~K}$ & $202 \mathrm{~K}$ & $170 \mathrm{~K}$ & $138 \mathrm{~K}$ & $119 \mathrm{~K}$ \\
\hline $\mathrm{A}$ & & & & & & \\
$p$ & 0.3409 & 0.3351 & 0.3354 & 0.3362 & 0.3362 & 0.3359 & 0.3356 \\
$q$ & 0 & 0 & 0 & -0.0014 & -0.0026 & -0.0069 & -0.0102 \\
$r$ & 0.4901 & 0.9422 & 0.9421 & 0.9418 & 0.9418 & 0.9419 & 0.9419 \\
$s$ & -0.011 & -0.012 & 0.002 & 0 & 0 & 0 & 0 \\
Sigma & 0.008 & 0.004 & 0.012 & 0.003 & 0.004 & 0.004 & 0.004 \\
TCNB & & & & & & & \\
$p$ & 0.3074 & 0.3054 & 0.3038 & 0.3037 & 0.3024 & 0.3007 & 0.2996 \\
$q$ & 0 & 0 & 0 & 0.0221 & 0.0300 & 0.0324 & 0.0322 \\
$r$ & 0.9516 & 0.9522 & 0.9528 & 0.9525 & 0.9527 & 0.9532 & 0.9535 \\
$s$ & 0.3476 & 3.452 & 3.461 & 3.457 & 3.450 & 3.343 & 3.430 \\
Sigma & 0.010 & 0.009 & 0.003 & 0.005 & 0.009 & 0.011 & 0.013 \\
$a$ & $2.03^{\circ}$ & $1.80^{\circ}$ & $1.92^{\circ}$ & $2.37^{\circ}$ & $2.76^{\circ}$ & $3.09^{\circ}$ & $3.25^{\circ}$ \\
\hline
\end{tabular}

(b). Least-squares lines of the form: $i=a_{1}+b_{i} t, j=a_{j}+b_{j} t$ and $k=a_{k}+b_{k} t$. All nonhydrogen atoms of the $A$ and TCNB molecules were used to determine the least-squares lines.

\begin{tabular}{|c|c|c|c|c|c|c|c|}
\hline \multicolumn{8}{|l|}{$A$} \\
\hline$a_{i}$ & -0.0690 & -0.0722 & -0.0314 & 0 & 0 & 0 & 0 \\
\hline$b_{i}$ & 0.9401 & 0.9422 & 0.9421 & 0.9382 & 0.9351 & 0.9341 & 0.9336 \\
\hline$a_{j}$ & 0 & 0 & 0 & 0 & 0 & 0 & 0 \\
\hline$b_{j}$ & 0 & 0 & 0 & 0.0883 & 0.1195 & 0.1306 & 0.1368 \\
\hline$a_{k}$ & 0.0136 & 0.0128 & 0.0148 & 0 & 0 & 0 & 0 \\
\hline$b_{k}$ & -0.3409 & -0.3351 & -0.3354 & -0.3348 & -0.3335 & -0.3322 & -0.3312 \\
\hline \multicolumn{8}{|c|}{ TCNB } \\
\hline$a_{i}$ & -0.2042 & -0.2175 & -0.1832 & -0.1749 & -0.1799 & -0.1894 & -0.1905 \\
\hline$b_{i}$ & 0.9516 & 0.9522 & 0.9527 & 0.9525 & 0.9527 & 0.9532 & 0.9535 \\
\hline$a_{j}$ & 0 & 0 & 0 & 0 & 0 & 0 & 0 \\
\hline$b_{j}$ & 0 & 0 & 0 & 0.0157 & 0.0279 & 0.0241 & 0.0246 \\
\hline$a_{k}$ & 0.3719 & 0.3695 & 0.3691 & 0.3686 & 0.3679 & 0.3662 & 0.3657 \\
\hline$b_{k}$ & -0.3074 & -0.3054 & -0.3038 & -0.3041 & -0.3031 & -0.3015 & -0.3004 \\
\hline$b$ & $2.03^{\circ}$ & $1.80^{\circ}$ & $1.92^{\circ}$ & $4.59^{\circ}$ & $6.00^{\circ}$ & $6.45^{\circ}$ & $6.77^{\circ}$ \\
\hline
\end{tabular}

${ }^{8}$ Angle between plane normal of $A$ and TCNB. ${ }^{b}$ Angle between the long axes of $A$ and TCNB. 
sitions of atoms $\mathrm{C} 4-\mathrm{C} 4 M$ or $\mathrm{C} 7-\mathrm{C} 7 M$. It is therefore appropriate to describe the $\mathrm{A}$ molecules as dynamically disordered characterized by libration about the molecular symmetry axis perpendicular to the plane of the molecule. In contrast to this observation, the electron density map for the donor at $202 \mathrm{~K}$, a temperature just below $T_{p}$, displays clear resolution of atom pairs C4$\mathrm{C} 4 I$ and $\mathrm{C} 7-\mathrm{C} 7 I$ (the change in space group symmetry is indicated by the change in labels: $M=$ mirror operator, $I=$ inversion center). There is, however, still considerable libration about the same molecular symmetry axis. As the temperature is lowered, there is a systematic improvement in the resolution of these atoms. Thus the double potentials describing the low temperature phase can be further characterized as being broad near $T_{p}$ but narrowing dramatically as the temperature is lowered.

The electron density plots for the TCNB molecule show a steady increase in electron density throughout the temperature range studied. Based on this information alone, a single well potential function would be indicated; however, the orientational behavior of the TCNB molecules in the low temperature phase, which will be described in the next section, may require a double well potential description. The nature of the potential functions will be examined further in the next section.

Rigid body thermal motion analysis ${ }^{13}$ of the structure at each temperature provides a useful, though probably not very accurate, estimate of the magnitude of libration about each of three principal axes that are approximately parallel to the molecular symmetry axes. Figure 3 displays the magnitudes of libration $\omega$ about each of these axes. Three features of the figure merit comment: (1) there is an apparently linear relationship between $T$ and $\omega$; (2) the relationship between $T$ and $\omega$ about the plane normal axis for the A molecule displays a different slope in the room temperature and low temperature phases; and (3) the temperature dependence of $\omega$ about the respective intraplane molecular axes is very similar for the A and TCNB molecules. The last observation carries the implication of concerted motion between donor and acceptor in both phases. It should also be pointed out that, in contrast to the A molecule, the largest libration for the TCNB molecule is not about its plane normal but about but about its intraplane long axis.

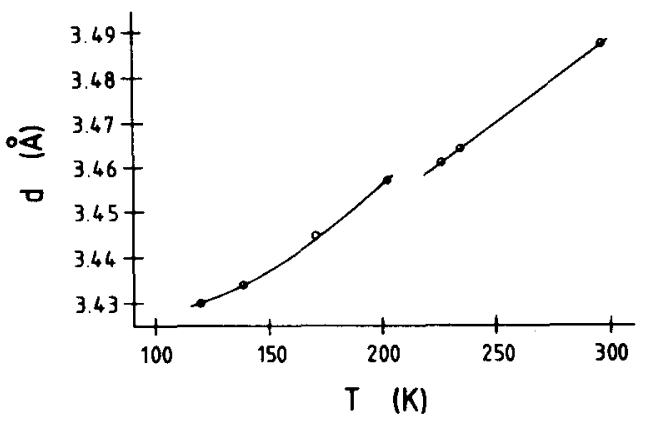

FIG. 5. The spacing between the A and TCNB planes within the CT stacks as a function of temperature. The estimated standard deviations are less than or equal to $0.005 \AA$.
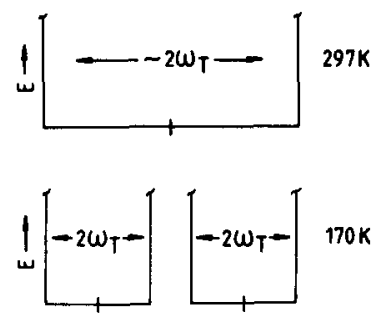

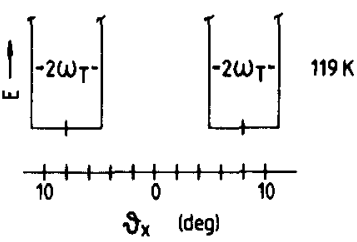

(a)

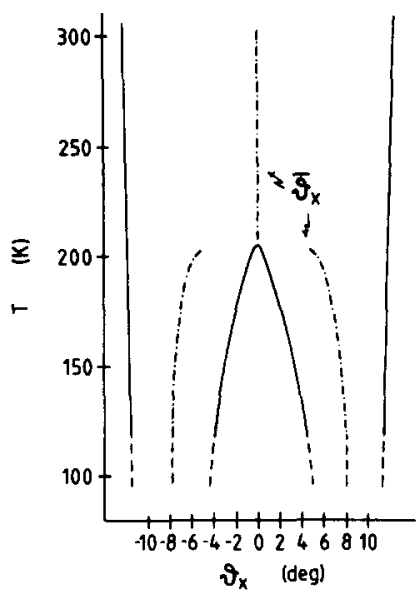

(b)
FIG. 6. The schematic characterization of the potential diagrams for the donor molecules in A: TCNB as a function of the orientation of the $x$ axis and libration about the $z$ axis. Part (a) depicts the relative breadth of the single and double well potentials at three temperatures and illustrates the temperature dependent angular separation of the midpoints of the double wells in the low temperature phase. Part (b) depicts these properties as a function of temperature. The latter may be regarded as displaying the temperature dependency of the boundaries of the double well potentials and their merging into a broad single well potential for the room temperature phase.

\section{Characterization of the low temperature phase of A:TCNB}

The crystal structure determinations of the A:TCNB complex at four temperatures below the phase transition at $206 \mathrm{~K}$ have provided data for direct characterization of the temperature dependent changes in the orientation of donor and acceptor molecules. These results confirm the quantitative results from spectroscopic measurements with respect to the reorientation of the long molecular axes of the donor but not the interpretation of the structural changes giving rise to them. ${ }^{3,4,6}$

As expected, the most dramatic manifestation of the temperature dependency of the structure of the low temperature phase is provided by the orientation of the $A$ molecules. As communicated earlier, ${ }^{9}$ all A molecules within a stack are related by pure translational symmetry and are therefore crystallographically identical. The glide plane permits orientational differences between symmetry related stacks. Assuming that the donor and acceptor molecules are planar (a crystallographically supported assumption), the differences in the orientation of symmetry related molecules can be characterized by two mean order parameters $\bar{\vartheta}_{x}$ and $\bar{\vartheta}_{z}$ where $\bar{\vartheta}_{x}$ is one-half the angle between the long molecular symmetry axes of related molecules and $\bar{\vartheta}_{z}$ is one-half the angle between their plane normais; experimentally determined values are presented in Fig. 4 . It is clear that the predominant change is in the orientation of the long axes of the $A$ molecules and that the magnitude of said change is in agreement with that determined by Möwald and co-workers $\mathrm{s}^{3,4}$ for $\bar{\vartheta}_{x}$. The definition of $\bar{\vartheta}_{z}$ here is more general than that of Erdle and Möwald ${ }^{4}$ in that the 


\section{4}
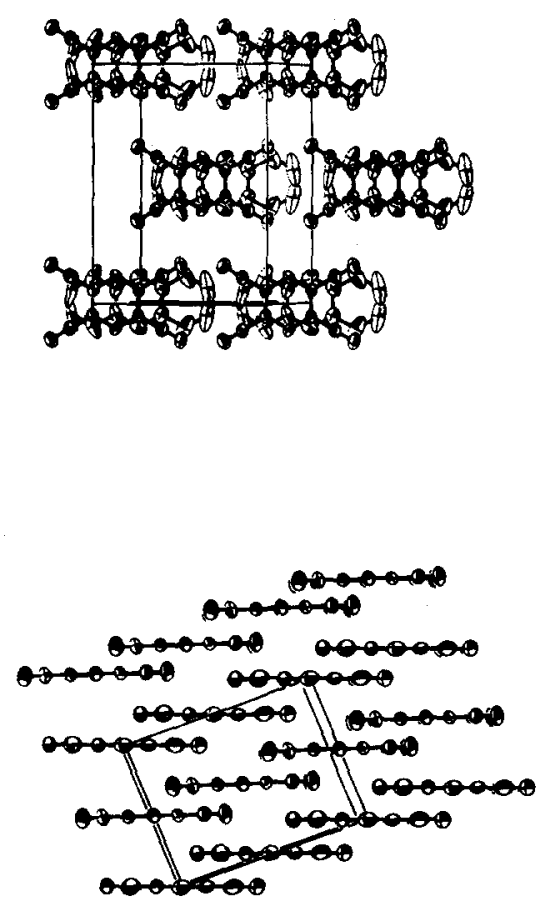

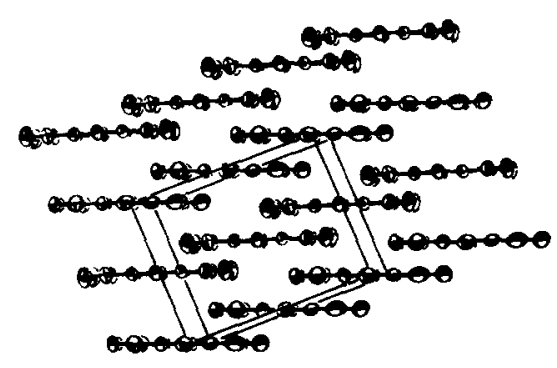

FIG. 7. Stereoscopic packing diagrams of A : TCNB at $297 \mathrm{~K}$. The upper stereo pair, (a), displays the packing viewed down a plane normal; the origin of the unit cell is at the lower rear left; the $b$ axis is vertical, the $a$ axis horizontal with a small out of paper component, and the $c$ axis is out of page with a minor horizontal component in the opposite direction as $a$. The lower pair displays the structure rotated by $90^{\circ}$ about the horizontal (parallel to the long axis of the molecules). The nearly coaxial donor and acceptor molecules (in the direction of the long molecular axis) are in translation related stacks. Stacks in the foreground are symmetry related to those in the background. Each stack consists of (from bottom to top) the sequence A : TCNB : A : TCNB. latter authors considered only a rotation about the long axis $x$ as the source of reorientation of molecular symmetry axis $z$, whereas the angle between the plane normals is describable by rotation about the general vector defined by the intersection of the planes of symmetry related molecules. The magnitude of $\bar{\vartheta}_{z}$ from this study is considerably smaller than that from the ESR study ${ }^{4}$ indicating that a compensatory reorientation, which may be viewed as a rotation component about the short molecular symmetry axis $y$, must be present. Quantitative data describing the orientation of the $x$ and $z$ axes in the room temperature phase and in the specific examples of the low temperature phase are presented in Table IV.

The crystal structure analyses also reveal orientational changes for the acceptor molecules. The mean order parameters for TCNB, Fig. 4, contrast with those for $A$ in that $\bar{\vartheta}_{z}$ is of comparable magnitude (slightly larger) with $\bar{\vartheta}_{x}$ and in the simila rity of the temperature dependence of $\bar{\vartheta}_{x}$ and $\bar{\vartheta}_{g}$. Unexpectedly, the changes in orientation (relative to the room temperature phase) of the plane normals for A and TCNB are in opposite directions for molecules in the same stack whereas those for the respective $x$ axes are in the same direction.

The probable reason for the changes in orientation is a drawing together of the donor and acceptor molecules, Fig. 5. The decrease in interplanar spacing that results is clearly crystallographically significant $(\sim 20 \sigma)$. There is a clear discontinuity in the relationship between the donor-acceptor interplanar spacing and temperature at the phase transition and a difference in temperature dependency in the room temperature and low temperature phases. It is probable that the nonlinearity of the curve below the phase transition represents an entropy contribution since the librational freedom changes dramatically with temperature.

It was concluded in the previous section that double well potential functions were necessary to describe the librational properties of the donor and most likely the ac ceptor molecules. The electron density plots indicate that a much broader potential function is required to account for the libration of the donor molecules than for the acceptors. The temperature dependence of the libration of the donor coupled with the $\bar{\vartheta}_{x}$ parameter thereof imply a temperature dependent series of double well potentials of the type presented in Fig. 6(a). As depicted therein, the the potentials merge as a result of increasing breadth and decreasing separation to give a broad single well potential that describes the room temperature phase. Figure $6(\mathrm{~b})$ displays a schematic representation of this process; it displays the boundaries of the potential wells as a function of temperature. The diagram was constructed, as illustrated in Fig. 6(a), to be consistent with both the angular separation of the long axes of symmetry related A molecules $\left(2 \cdot \bar{\vartheta}_{x}\right)$ and the magnitude of the libration about the $z$ axis, $2 \omega$. At temperatures above $\sim 190 \mathrm{~K}$, the magnitude of libration is larger than $\bar{y}_{x}$.

No effort has been made to construct a similar diagram for TCNB since the acceptors do not display comparable anisotropy in their libration; the libration behavior, Fig. 3, is consistent with coupled motion with the donors. The reorientation of the TCNB molecules is such that it very likely represents an accommodation of 

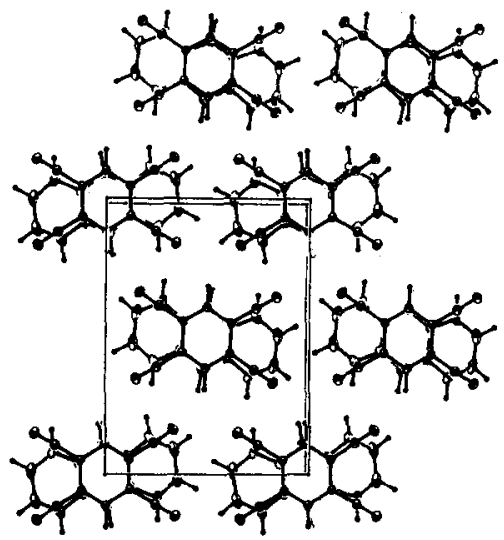
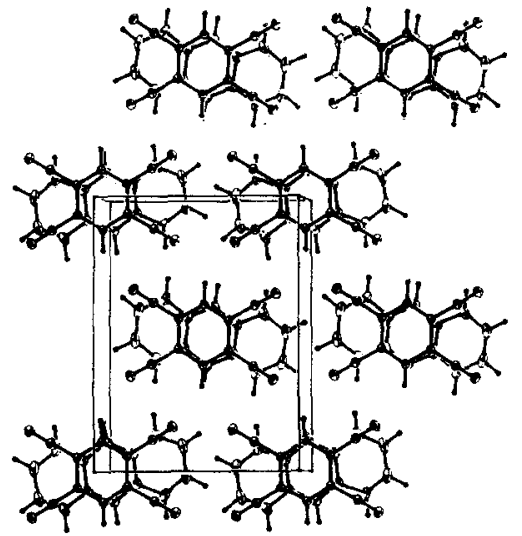

FIG. 8. Packing diagram for $A$ :TCNB at $119 \mathrm{~K}$. This figure differs from Fig. 7(a) slightly in that projection is on the $a b$ plane rather than on the plane of one on the acceptor molecules. The hydrogen atoms are also included in this figure. The origin of the depicted unit cell is at the back lower left. The $b$ axis is vertical, the $a$ axis horizontal, and the $c$ axis out of page. The marked reorientation of the $A$ molecules is apparent in comparison of Figs. 7 and 8. that of the A molecules in a manner that allows closer contact between the components of the charge transfer complex in the low temperature phase, Fig. 5.

\section{Crystal packing}

The crystal structure of A : TCNB has been characterized $^{3,4, B}$ as consisting of separate planes of $A$ and TCNB molecules. This description is not rigorously

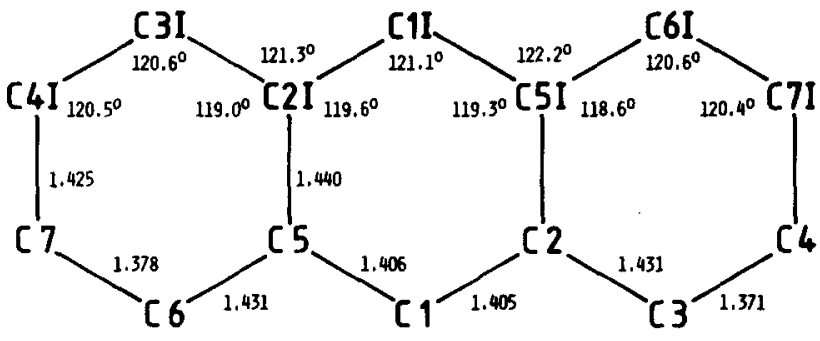

(a)

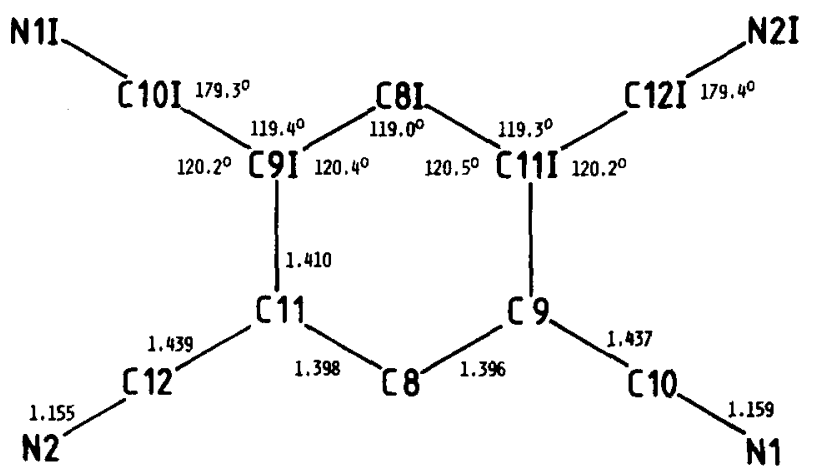

(b)

FIG. 9. Bonding geometry for $A$ and TCNB derived from the crystal structure of $A: T C N B$ at $119 \mathrm{~K}$. All values are corrected for rigid body thermal motion; typical corrections are on the order of $0.003 \AA$. The estimated standard deivations in bond distances are less than $0.002 \AA$ and in bond angles less than $0.1^{\circ}$. Suitable comparison for the A molecules can be found in the report of the structure of the charge transfer complex between $\mathrm{A}$ and pyromellitic dianbydride ${ }^{15}$ and for TCNB in the report of the structures of uncomplexed $\mathrm{TCNB}^{16}$ and of tetracyanoethylene. ${ }^{17}$ correct in that the molecular planes are not coplanar with the planes defined by the midpoints of the molecules; the latter apparently define the planes alluded to. Figures 7 and 8 present stereoscopic packing diagrams for the structures at 297 and $119 \mathrm{~K}$ in similar orientations. These diagrams demonstrate that $\mathrm{A}$ and TCNB molecules from adjacent stacks are approximately coaxial (long molecular axes) in the (102) direction, whereas stacks in the (010) direction display planes parallel

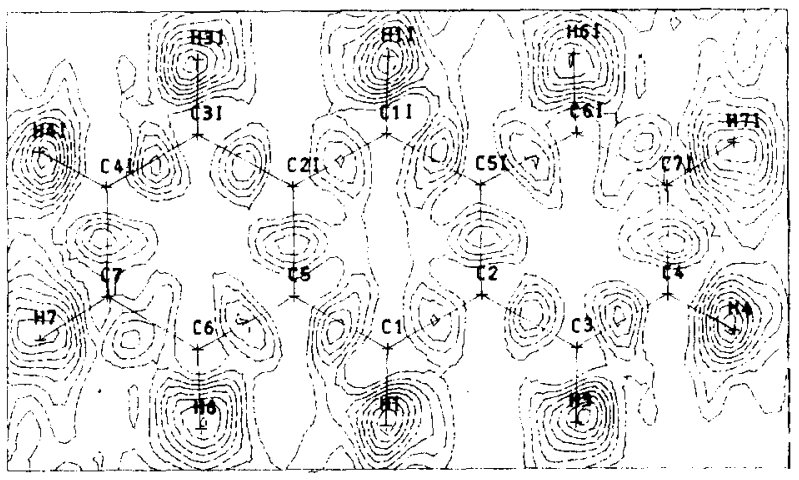

(a)

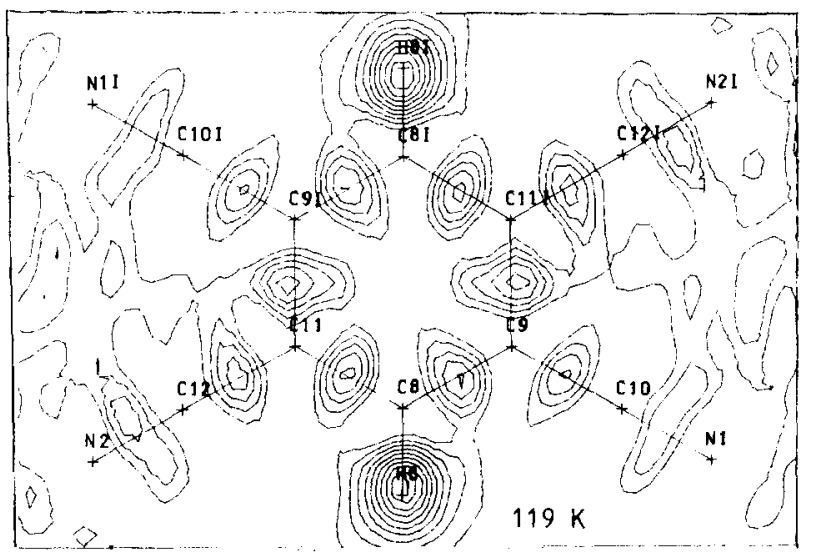

(b)

FIG. 10. Difference electron density maps for A and TCNB for the $119 \mathrm{~K}$ data set. $\mathrm{H}$ atoms have been removed for the structure factor calculation to illustrate their electron density and provide comparison for the electron density remaining between bonded $\mathrm{C}$ and $\mathrm{N}$ atoms. Each contour is 0.1 electron $\AA^{-3}$. 
thereto but displaced by one-half the thickness of the CT complex.

Figure 7 also provides insight into the likely reason why the TCNB molecules do not show high thermal libration. The heteroatomic nature of the cyano moieties gives rise to an intramolecular dipole oriented along the $\mathrm{C} \equiv \mathrm{N}$ bond. The disposition of the TCNB molecules in the crystal is nearly ideal for the interaction of two oppositely oriented dipoles for each of the cyano moieties. It is also noteworthy that the A molecules coplanar with a given TCNB molecule librate between the $\delta^{-}$charged $\mathrm{N}$ atoms of the acceptor. This arrangement is intriguing with respect to the disposition of charge in the excited state.

To complete characterization of the structure, bond distances and bond angles for the donor and acceptor molecules derived from the structure at $119 \mathrm{~K}$ are presented in Fig. 9. A table of intermolecular contact distances at each temperature has been deposited. ${ }^{11} \mathrm{~A}$ final difference Fourier map for the mean planes of the $A$ and TCNB molecules is presented in Fig. 10. The electron density of the $\mathrm{H}$ atoms and the residual bonding electron density is clearly observable therein.

\section{ACKNOWLEDGMENT}

Thanks are due to Dr. J. von Schütz for the crystal of A : TCNB used in this study and for helpful discussion in the course of the analysis of the results. The assistance of $\mathrm{Mr}$. R. Prewo in the preparation of the electron density plots is also most gratefully acknowledged.

${ }^{1}$ A. M. Ponte Goncalves, Chem. Phys. 19, 397 (1977).

${ }^{2}$ H. M. Vyas and A. M. Ponte Goncalves, Chem. Phys. Lett.
51,556 (1977).

${ }^{3}$ H. Möwald, E. Erdle, and A. Thaer, Chem. Phys. 27, 79 (1978).

${ }^{4}$ E. Erdle and H. Möwald, Solid State Commun. 26, 327 (1978).

${ }^{5}$ W. A uch, W. Steudle, J. U. van Schütz, and H. C. Wolf, Phys. Status Solidi A 49, 563 (1978).

${ }^{6}$ H. Möwald and A. Thaer, Phys. Status Solidi A 50, 131 (1978).

${ }^{7} \mathrm{~W}$. Steudle and J. U. von Schütz, Abstracts of the International Conference on Luminescence, Paris, 1978, p. 74; Proceedings of the 1978 International Conference on Luminescence, Paris, 17-21 July, 1978; J. Lumin. 18/19, 191 (1979).

${ }^{8}$ H. Tsuchiya, F. Marumo, and Y. Saito, Acta Crystallogr. Sect. B 28, 1935 (1972).

${ }^{9}$ J. J. Stezowski, J. Phys. Chem. 83, 550 (1979).

${ }^{10}$ J. M. Stewart, P. A. Machin, C. W. Dickinson, H. L. Ammon, H. Flack, and H. Heck, The XRAY System-Version 1976, Technical Report TR-446, Computer Science Center, University of Maryland, College Park, MD. Unless otherwise indicated, this program library was used for all computerized calculations.

11، See AIP document no. PAPS JCPSA-73-538-96 for 96 pages of material including calculated and observed structure factors for the structure at each temperature, anisotropic temperature factors for $\mathrm{C}$ and $\mathrm{N}$ atoms, fractional coordinates and isotropi temperature factors of $\mathbf{H}$ atoms in the low temperature phase, and intermolecular interatomic contact distances to $\sim 3.6 \AA$.

${ }^{12} J$. J. Stezowski, C. Bürvenich, and W. Voelter, Angew. Chem. Int. Ed. 18, 225 (1979).

${ }^{13}$ V. Schomaker and K. Trueblood, Acta Crystallogr. Sect. B 24, 63 (1968).

${ }^{14}$ Program JIMPLAN, A Oblique Plane Fourier Plotting Program, in which the slant plane Fourier transform routine of $B$. W. van de Waal has been incorporated by $N$. K. Hanson.

${ }^{15}$ B. E. Robertson and J. J. Stezowski, Acta Crystallogr. Sect. B 34, 3005 (1978).

${ }^{16} \mathrm{C}$. K. Prout and I. J. Tickle, J. Chem. Soc. Perkin II, $1978,520$.

${ }^{17}$ P. Becker, P. Coppens, and F. K. Ross, J. Am. Chem. Soc. 95, 7604 (1973).

${ }^{18}$ W. C. Hamilton, Acta Crystallogr. 18, 502 (1965). 